\title{
Atomic Structure of Extended Defects in GaAs-based Heterostructures
}

\author{
Abhinandan Gangopadhyay $^{1 *}$, Aymeric Maros ${ }^{2}$, Nikolai Faleev ${ }^{2}$, Richard R. King ${ }^{2}$ and David J. Smith ${ }^{3}$ \\ 1. School for Engineering of Matter, Transport and Energy, Arizona State University, Tempe, AZ \\ 2. School of Electrical, Computer and Energy Engineering, Arizona State University, Tempe, AZ \\ 3. Department of Physics, Arizona State University, Tempe, AZ \\ * Corresponding author: abhinandan.gangopadhyay@asu.edu
}

The atomic structure of defects is important to understand since this determines the relaxation kinetics in semiconductor heterostructures as well as electronic properties. Interactions with point defects/impurities, and other line defects, as well as reconstruction of the defect core to locally minimize the distortion field, create numerous possibilities for structural arrangements of the defects. Recent advances in aberration correction for the transmission electron microscope have led to the achievement of sub-angstrom resolution. Thus, atomically-resolved images of defects can be routinely obtained, providing an opportunity to characterize structural defects with greater detail. In this work, extended defects have been investigated using probe-corrected scanning transmission electron microscope (STEM), in a range of GaAs(001)-based epitaxial heterostructures grown by molecular beam epitaxy. The TEM samples were prepared using conventional mechanical polishing, dimpling and argon ionmilling at liquid-nitrogen temperature. A JEOL ARM-200F operated at $200 \mathrm{kV}$ was used for high-angle annular dark-field (HAADF)-STEM imaging. The probe convergence angle was $22 \mathrm{mrad}$, and the inner and outer angles for HAADF-STEM imaging were 90 and $150 \mathrm{mrad}$.

Strain relaxation in low-mismatched heterostructures was primarily carried out by dissociated $60^{\circ}$ dislocations. Figure 1(a) shows a HAADF-STEM image of a misfit dislocation at the film/substrate interface of an $\mathrm{In}_{0.08} \mathrm{Ga}_{0.92} \mathrm{As} / \mathrm{GaAs}$ heterostructure (misfit strain $\sim 0.6 \%$ ). Burgers circuit analysis identified a dissociated $60^{\circ}$ dislocation bounded by $30^{\circ}$ and $90^{\circ}$ Shockley partials at its two ends. The $30^{\circ}$ Shockley partials had a characteristic single atomic column at their core, indicating that these dislocations belonged to glide set. The leading $30^{\circ}$ partials of negative dislocations on intersecting glide planes often interacted to form Lomer-Cottrell locks with sessile $\boldsymbol{a} / 6<110\rangle$ stair-rod dislocations in compressively-strained heterostructures. Hirth locks with $a / 3<001>$ stair-rod dislocations were not observed as their formation would require a reaction between negative and positive dislocations, whereas only negative dislocations were created to relieve misfit strain in these compressively-strained heterostructures. The density of perfect $60^{\circ}$ dislocations was much reduced compared to dissociated $60^{\circ}$ dislocations. Figure 1(b) shows a HAADF-STEM image of a perfect $60^{\circ}$ dislocation located close to the $\mathrm{In}_{0.08} \mathrm{Ga}_{0.92} \mathrm{As} / \mathrm{GaAs}$ interface. The extra-half plane has been marked by a red dashed line. The majority of the misfit dislocations in tensile-strained $\mathrm{GaAs}_{0.9} \mathrm{P}_{0.1} / \mathrm{GaAs}$ (misfit strain $\sim 0.4 \%$ ) heterostructures were dissociated $60^{\circ}$ dislocations bounded by $90^{\circ}$ and $30^{\circ}$ partials. Figure 1(c) shows a HAADF-STEM image of a dissociated $60^{\circ}$ dislocation at the $\mathrm{GaAs}_{0.9} \mathrm{P}_{0.1} / \mathrm{GaAs}$ interface. Although formation of $a / 3<110>$ stair-rod dislocations by the interaction of leading $90^{\circ}$ partials of positive misfit dislocations on intersecting $\{111\}$ glide planes is energetically favorable, such defect configurations were not observed. Figure 2(a) shows a bright-field STEM image of a growth defect extending all the way through the epitaxial film to its top surface. This defect originated at about $142 \mathrm{~nm}$ underneath the film/substrate interface in the GaAs buffer layer. Figure 2(b) shows a HAADF-STEM image illustrating that the defect started as an intrinsic stacking fault but transformed into a coherent twin upon operation of Shockley partial dislocations on adjacent $\{111\}$ planes. 
Strain in high-mismatched heterostructures can be spontaneously released by the formation of an array of $90^{\circ}$ Lomer dislocaions at the film/substrate interface. The atomic configuration of these dislocation cores in technologically important systems such as GaSb/GaAs(001) (misfit strain 7.8\%) is not wellunderstood. Further investigations are ongoing to unravel the core structure of Lomer dislocations at $\mathrm{GaSb} / \mathrm{GaAs}$ hetero-interfaces. [1]

\section{References:}

[1] This work was supported by the National Science Foundation and the Department of Energy under NSF CA No. EEC-1041895. The authors acknowledge the use of facilities within the John M. Cowley Center for High Resolution Electron Microscopy at Arizona State University.
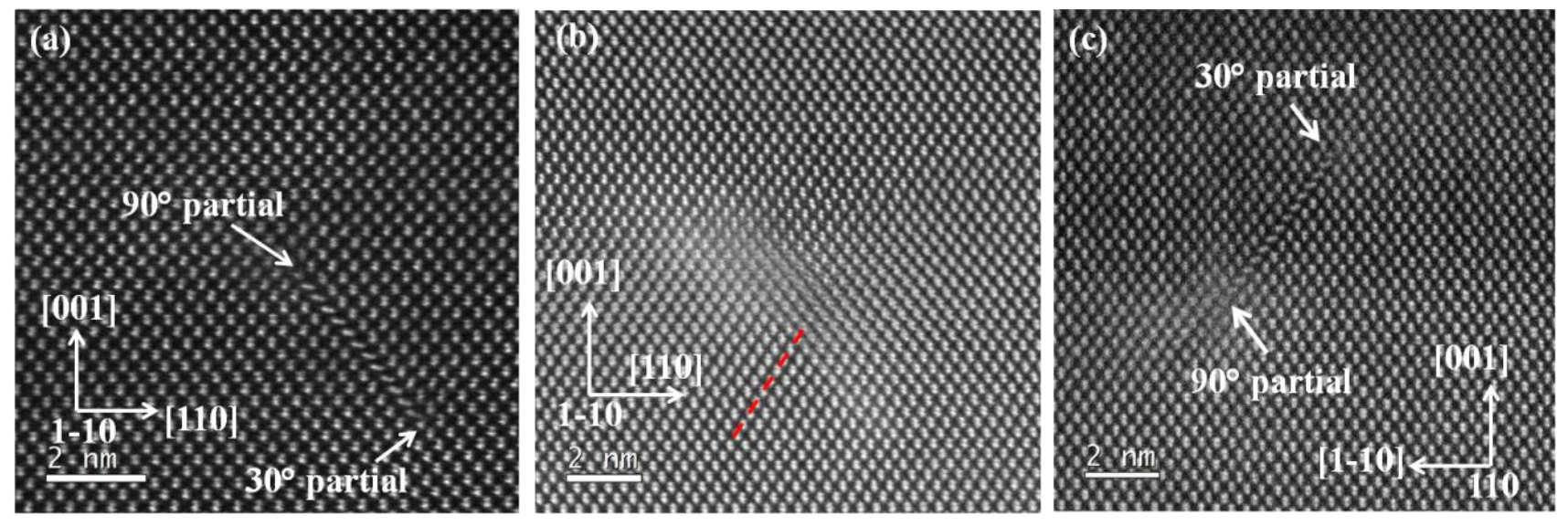

Figure 1. Aberration-corrected HAADF-STEM images: (a) Interfacial dissociated $60^{\circ}$ dislocation in $\mathrm{In}_{0.08} \mathrm{Ga}_{0.92} \mathrm{As} / \mathrm{GaAs}(001)$ heterostructure; (b) Perfect $60^{\circ}$ dislocation in $\operatorname{In}_{0.08} \mathrm{Ga}_{0.92} \mathrm{As} / \mathrm{GaAs}(001)$ heterostructure; and (c) Interfacial dissociated $60^{\circ}$ dislocation in $\mathrm{GaAs}_{0.9} \mathrm{P}_{0.1} / \mathrm{GaAs}(001)$ heterostructure.
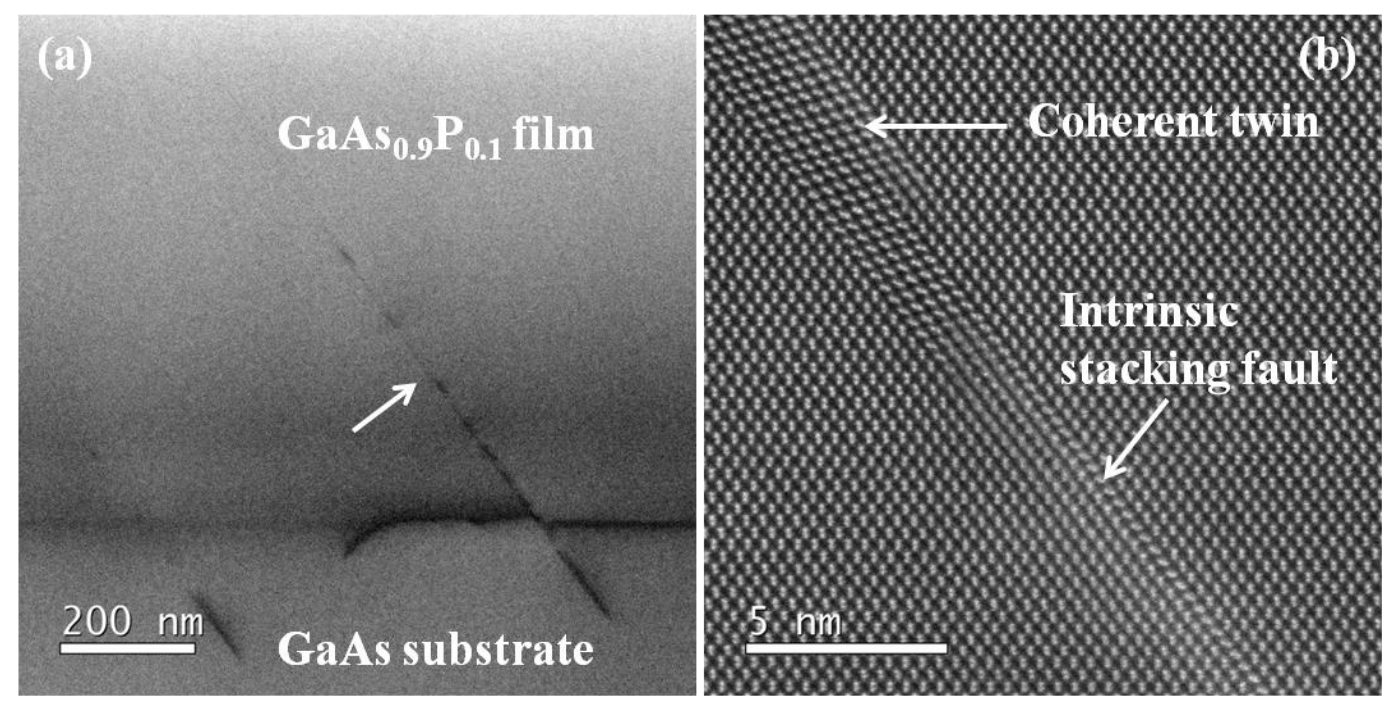

Figure 2. (a) Bright-field STEM image showing defect (indicated by white arrow) that extends through the entire film of $\mathrm{GaAs}_{0.9} \mathrm{P}_{0.1} / \mathrm{GaAs}$ heterostructure; (b) Atomic-resolution HAADF-STEM image reveals that the defect nucleates as intrinsic stacking fault, but transforms into a twin fault. 